\title{
Reflets
}

Revue ontaroise d'intervention sociale et communautaire

\section{La Maison Verte : un cas réussi de développement économique communautaire}

\section{Mufaume Kihumbi}

Volume 5, numéro 1, printemps 1999

Pratiques et développement économique communautaire

URI : https://id.erudit.org/iderudit/026258ar

DOI : https://doi.org/10.7202/026258ar

Aller au sommaire du numéro

Éditeur(s)

Reflets : Revue ontaroise d'intervention sociale et communautaire

ISSN

1203-4576 (imprimé)

1712-8498 (numérique)

Découvrir la revue

Citer cet article

Kihumbi, M. (1999). La Maison Verte : un cas réussi de développement économique communautaire. Reflets, 5(1), 176-183.

https://doi.org/10.7202/026258ar

Tous droits réservés (C) Reflets : Revue ontaroise d'intervention sociale et communautaire, 1999
Ce document est protégé par la loi sur le droit d'auteur. L'utilisation des services d'Érudit (y compris la reproduction) est assujettie à sa politique d'utilisation que vous pouvez consulter en ligne.

https://apropos.erudit.org/fr/usagers/politique-dutilisation/ 


\title{
La Maison Verte: un cas réussi de développement économique communautaire ${ }^{1}$
}

\author{
Mufaume Kihumbi \\ Étudiant, maîtrise en sociologie, Université Laurentienne
}

La Maison Verte, établie à Hearst depuis 1982, est un exemple typique d'un organisme de développement économique communautaire (DÉC) dans le Nord-Est de l'Ontario. Établie grâce à l'appui financier des gouvernements fédéral et provincial ainsi que d'un groupe important d'investisseurs locaux, la Maison Verte a pour objectif, depuis ses débuts, de procurer des emplois aux femmes et de s'enraciner dans sa communauté pour la faire bénéficier de ses activités et retombées économiques. Quinze ans après son établissement, cette entreprise de DÉC emploie neuf travailleuses à plein temps et vingt autres travailleuses saisonnières.

Il s'agit d'une entreprise communautaire dont la principale production consiste en des plants de reboisement. Elle produit trois espèces d'arbres, l'épinette noire, l'épinette blanche et le pin gris. Située à quelques kilomètres de la ville de Hearst, elle occupe un complexe de 50000 pieds carrés de serres jumelées, 46000 pieds d'ombrières et 57000 pieds carrés de serres d'été. L'entreprise vend actuellement 6400000 plants et, poursuivant sa politique de diversification des produits adoptée en 1992 suite à une coupure au budget du reboisement, fait pousser une grande variété de fleurs annuelles, de vivaces et de plants de légumes tels la tomate, le poivron et le concombre. 


\section{Ses origines}

La création de la Maison Verte est indissociable de l'histoire de l'organisme. Parmi-elles, une association à but non lucratif formée par les femmes de la région de Hearst, dans le but de créer des emplois pour les femmes ainsi que de faire la promotion de l'autonomie et du bien-être des femmes de la région.

L'élément déclencheur de la création de l'association fut le colloque Au Féminin II, tenu le 24 octobre 1979 et organisé par l'association Franco-femmes. Le colloque portait sur certains problèmes et thèmes reliés à la situation des femmes. Dans un atelier sur la famille monoparentale, une fonctionnaire du ministère d'Emploi et Immigration Canada à Timmins discuta d'un programme fédéral, PACLE (Programme d'assistance à la création locale d'emploi), qui procurait de l'aide aux membres éligibles d'un groupe pour se créer des emplois. Le programme s'adressait aux personnes difficilement employables et leur permettait d'acquérir leur propre entreprise et, par conséquent, une certaine indépendance. Il défrayait les coûts de capitaux, l'entraînement et la formation dans la nouvelle fonction ainsi que les salaires pour les premiers trois ans.

Par définition, plusieurs femmes qui se retrouvent seules avec leurs enfants, qui n'ont aucune formation académique ni technique et qui dépendent d'assistance sociale ou d'emplois précaires, tombent dans cette catégorie de personnes difficilement employables.

Tel est le cas, par exemple, de Nicole Roy, l'une des initiatrices de Parmi-elles. Suite à l'atelier, elle réussit à réunir un groupe de femmes monoparentales. À ses débuts le groupe prend le nom de Une pour Deux et se fixe pour objectifs l'identification de problèmes communs aux membres et surtout la recherche de solutions. C'est en discutant de solutions possibles que le groupe songe au programme fédéral PACLE.

Un peu plus tard, pour permettre à d'autres femmes qui n'étaient pas mono-parentales de se joindre au groupe, et évidemment parce que plusieurs de ces femmes étaient intéressées 
au projet, on change le nom du groupe à Parmi-elles. Il s'agissait en fin de compte de plus qu'un simple changement de nom. En effet, on décide aussi de modifier les critères d'adhésion pour admettre toute personne qui s'intéresse au projet et qui veut travailler à sa réalisation. Certaines employées du bureau de l'assurance-chômage ont même accepté de siéger au conseil d'administration.

\section{La création de l'entreprise}

L'histoire de la Maison Verte en tant qu'entreprise commerciale remonte à 1981, soit un an et demi après la création de l'association Parmi-elles. Pour répondre aux exigences du programme fédéral de création d'emplois PACLE, l'association procède à l'étude de certains projets, dont une pâtisserie et la production d'huiles de conifere pour la fabrication de produits pharmaceutiques. En juillet 1981, l'association entreprend l'étude d'un projet de construction de serres dans le cadre d'un programme offert par le ministère des Richesses naturelles. En 1982, grâce à un soutien financier additionnel du ministère et de soixante dix-sept (77) investisseurs locaux, le projet se concrétisa par une entente avec le gouvernement pour la construction d'un complexe de serres. C'est à ce moment que l'association Parmi-elles, devenue entreprise commerciale, s'incorpore sous le nom de La Maison Verte et procède ainsi à élargir ses objectifs.

En mai 1983, après la construction de serres, l'entreprise entre en production, soit celle de 2000000 de plants d'épinette noire et de pin gris. En vertu d'une entente avec le ministère des Richesses naturelles, cette production annuelle doit durer pour une période de cinq ans.

En 1987, Parmi-elles franchit une étape importante: elle rachète les actions privilégiées au coût de $273000 \$$, devenant ainsi seule propriétaire de l'entreprise. La même année, elle signe un contrat de cinq ans avec la Hearst Forest Management Inc., augmentant ainsi la production annuelle à 4000000 de plants et 
forçant la construction de cinq nouvelles serres et d'un nouveau système de chauffage aux granules Energex.

\section{Le soutien du milieu}

C'est grâce à l'initiative des organisateurs et à l'implication de la communauté de Hearst que le projet s'est concrétisé. Les femmes du milieu étaient de plus en plus intéressées à se joindre au groupe; les personnes-ressources et les investisseurs locaux voulaient également voir le projet se réaliser. Par personnes ressources, on veut dire les personnes du milieu, fonctionnaires ou gens d'affaires, susceptibles de fournir l'information et à constituer un réseau de contacts. Le Centre d'emploi est un exemple d'une agence gouvernementale où l'on a trouvé des personnes ressources. La Corporation de développement économique de Hearst a coordonné la campagne, invitant divers groupes à appuyer financièrement le projet.

L'association Parmi-elles a fait une offre d'actions privilégiées, d'une valeur de 100 \$ l'unité, auprès des investisseurs locaux. Ces derniers ont réagi favorablement, ce qui a permis de prélever une somme de 275000 \$. Les actions privilégiées, dont les propriétaires bénéficiaient du crédit d'impôt sur les dividendes, rapportaient un dividende cumulatif de $15 \%$ par an. Les investisseurs locaux constituaient $40 \%$ du conseil d'administration et Parmi-elles $60 \%$.

Par le biais du programme PACLE, le gouvernement fédéral a financé une grande partie des coûts de l'entreprise. Il s'est engagé à débourser 44000 \$ pour l'étude de faisabilité et à défrayer pendant trois ans le salaire des employés pour un montant maximum de $275000 \$$. Pour sa part, le gouvernement provincial a accepté de défrayer la moitié des fonds requis pour la construction, soit une somme de 200000 \$ et d'acheter la production des serres pour cinq ans.

Ainsi, forte de l'appui du milieu et animée par l'objectif de créer des emplois principalement pour des femmes dans la région de Hearst, la MaisonVerte a réussi à répondre aux défis de départ. 


\section{Trois dimensions}

Cet organisme de DÉC a réussi, au cours des derniers 15 ans, au niveau de trois dimensions bien précises : économique, associative et territoriale.

\section{La dimension économique}

Fondée pour promouvoir l'autonomie et le bien-être des femmes dans la région de Hearst, La Maison Verte est un apport communautaire à la lutte contre la crise d'emplois en général. Elle offre des emplois aux femmes et participe au développement économique de la communauté de Hearst. L'entreprise a débuté avec sept employés dont six femmes et un homme. À l'heure actuelle, elle emploie neuf personnes à temps plein et une vingtaine de saisonnières. Ces emplois, stables et permanents, sont maintenant ouverts à toutes les femmes ainsi qu'aux étudiantes.

La main-d'oeuvre de l'entreprise est donc constituée de femmes qui, pour la majorité, travaillaient à temps partiel dans des entreprises de services et dont l'entrée sur le marché du travail stable et permanent était devenue chose très difficile, sinon impossible. Elles viennent à l'entreprise sans expérience et sans formation académique ou technique afin d'y chercher des connaissances qui leur permettent de comprendre le fonctionnement de l'entreprise et de se préparer à l'exercice de leur métier.

\section{La dimension associative}

La gestion de La Maison Verte est participative. On retrouve à sa tête un conseil d'administration au sein duquel les décisions sont prises collectivement. La participation des employées à la gestion et aux bénéfices de l'entreprise a contribué à développer un fort sentiment d'appartenance. Les employées sont continuellement informées quant à la gestion et au fonctionnement de l'entreprise. Ainsi, tous les vendredis, les employées se réunissent pour discuter et échanger des opinions et de l'information au sujet de l'entreprise. 
Depuis le début des années 1990, les employées participent aux bénéfices de La Maison Verte. C'est ainsi que $10 \%$ du total des bénéfices, avant impôt, sont répartis auprès des employées à la fin de l'année. Ces profits sont distribués à toutes les employées tant à temps plein que saisonnières. Pour les employées à temps plein, la moitié des bénéfices reçus est placée dans un régime de pension (REÉR) et l'autre moitié est donnée en argent. Quant aux employées saisonnières, les bénéfices sont répartis selon le nombre d'heures ( le minimum étant de 300 heures par an pour accéder au droit de participation) et cette répartition se fait de la façon suivante: $50 \%$ sur la base du salaire et l'autre $50 \%$ sur la base de l'ancienneté.

Le sentiment d'appartenance est fort; il s'exprime notamment par ce que La Maison Verte a appelé «le partage de travail». En 1992, lors des coupures au budget du reboisement, l'entreprise a décidé de mettre en vigueur un régime de partage de travail.Au lieu de congédier certaines employées (il aurait fallu en congédier au moins trois), elle procéda à la diminution d'heures de travail pour toutes les employées.

La Maison Verte est une entreprise franco-ontarienne. La langue du fonctionnement ainsi que de la communication interne est le français.Toutefois, le personnel clé est bilingue car les clients et les fournisseurs sont en majorité de langue anglaise.

\section{La dimension territoriale}

En ce qui a trait à sa contribution à l'économie locale, La Maison Verte a su s'enraciner dans le milieu, ce qui s'explique par son marché, son implication dans le développement de la région et son partenariat avec les compagnies locales. Elle écoule sa production sur place et ses principaux clients sont les compagnies forestières locales, la Hearst Forest Management Inc. et la QUNO. La Hearst Forest Management est également un grand partenaire qui l'assiste en matière technique: on échange l'information sur le type de plants préférés par la compagnie, sur la taille des plants requis.

Vu la dépendance de l'économie et de la qualité de vie de la population du Nord de l'Ontario sur les ressources naturelles, principalement forestières, il faut s'assurer que les générations 
futures soient en mesure de jouir de cette qualité de vie en remplaçant la richesse exploitée. La Maison Verte s'enracine de plus en plus dans le milieu pendant que sa participation au développement économique de ce dernier prend de l'ampleur.

L'entreprise a développé l'infrastructure et l'expertise nécessaires pour répondre aux besoins du marché local afin d'être en mesure d'offrir des produits de meilleure qualité. C'est ainsi qu'actuellement, elle fait partie d'une coopérative de recherche forestière dont le siège social est situé à l'Université Lakehead de Thunder Bay, qui finance des projets dans le but de permettre de développer des techniques de production telles que la diversification des produits.

La demande de plants par Hearst et la région ne cesse d'augmenter. Avant, elle était évaluée annuellement aux environs de 3000000 de plants, mais depuis l'entente signée entre les compagnies de bois et l'Entente de la gestion forestière, la demande a augmenté. Cette entente a permis à La MaisonVerte d'augmenter sa production à 6400000 arbres.

L'Entente de la gestion forestière est une initiative des entreprises forestières locales qui se sont réunies pour répondre au retrait du gouvernement provincial en matière de reboisement. Avant, c'était au ministère des Richesses naturelles qu'incombait la tâche du reboisement, mais maintenant, le gouvernement a remis cette responsabilité aux compagnies locales et privées. Dans l'esprit de cet accord, La MaisonVerte traite avec la Hearst Forest Management Inc.

\section{Parmi-elles et la Maison Verte: deux mondes?}

L'un des prix à payer pour la réussite de cette entreprise, c'est l'affaiblissement du rôle joué par l'association Parmi-elles car tous les efforts ont été mis dans la réalisation du projet de départ. Certains membres des premières heures ont abandonné l'association alors que d'autres - environ une dizaine, dont deux employées - ont continué d'y adhérer. 
Au nombre des solutions visant à consolider l'aspect associatif, Parmi-elles songe à créer une fondation à partir des bénéfices générés et réinvestis par La Maison Verte. Les fonds de cette fondation serviraient ainsi à la création de services, tels le financement de projets dont l'objectif serait de créer des emplois pour les femmes de la région. On espère ainsi que l'association continuera de jouer un rôle actif, comme elle le faisait auparavant, maintenant que le «bébé a grandi».

\section{Conclusion}

Note

La Maison verte est un bon exemple d'une entreprise de DÉC dont l'objectif est le développement de ses membres et de son milieu. La réussite de La Maison Verte est une réussite de la communauté de Hearst, en général, et des femmes organisatrices de l'initiative, en particulier. La Maison Verte participe non seulement au mieux-être des femmes de Hearst mais aussi à celui de la communauté toute entière. L'entreprise envisage de créer, en plus de la Fondation, un projet de recyclage pour ainsi s'impliquer dans la promotion de la qualité de l'environnement. Mais cette réussite ne va pas toujours de soi. Elle exige l'implication de tout le milieu, un sentiment d'appartenance très fort et une conviction sans faille. Ce sont là certains des facteurs et des conditions nécessaires pour redonner vie à sa communauté.

1. Mufaume Kihumbi (1997) (s. la dir. de Rachid Bagaoui), Le développement économique communautaire et la crise de l'emploi à Hearst: le cas de la Maison Verte, Mémoire de spécialisation, Département de sociologie, Université Laurentienne. 